\title{
ON ITERATIVE METHODS IN LINEAR DIFFERENTIAL EQUATIONS
}

\author{
BY \\ KENNETH S. MILLER
}

\section{Introduction.}

1.1. Statement of the problem. The problem we are interested in is that of estimating rates of convergence in solving differential equations by an iterative process. Suppose we have a differential equation and a method for computing a sequence of approximate solutions $u_{0}, u_{1}, \cdots, u_{k}, \cdots$ which satisfy the boundary conditions and have the property that $u_{k}$ approaches a solution as $k$ becomes infinite. Also, let us suppose that it is desired to have the solution such that the error (defined in some appropriate sense) be less than a certain prescribed quantity, $\delta$. The task we set ourselves is to determine how many times we must iterate the approximate solution to obtain the desired accuracy. In other words, we would like to say at the outset that after $k$ iterations the quantity $u_{k}$ will yield an error less than $\delta$ while the $u_{k-1}$ iterant will not yield the desired accuracy. We shall thus have shown that $k$ is the minimum number of operations that have to be performed. We consider this problem (formulated in $\$ 1.2$ ) for a certain class of differential systems.

1.2. Analytic formulation of the problem. We shall restrict ourselves to linear differential equations. Let $L$ be the linear differential operator

$$
L=\frac{d^{n}}{d x^{n}}+p_{1}(x) \frac{d^{n-1}}{d x^{n-1}}+\cdots+p_{n}(x) .
$$

where the $p_{i}(x)$ are real continuous functions in the closed finite interval $[0, c]$. Let

$$
U_{\alpha}(u) \equiv V_{\alpha}(u)+Z_{\alpha}(u)=C_{\alpha}, \quad \alpha=1,2, \cdots, n,
$$

where $V_{\alpha}(u)=\sum_{l=0}^{n-1} A_{\alpha i} u^{(i)}(0), Z_{\alpha}(u)=\sum_{i=0}^{n-1} B_{\alpha i} u^{(i)}(c), \alpha=1,2, \cdots, n$, be a set of nonhomogeneous boundary conditions such that the completely homogeneous differential system

$$
L u=0, \quad U_{\alpha}(u)=0, \quad \alpha=1,2, \cdots, n,
$$

is incompatible( ${ }^{(1)}$. The $A_{\alpha i}, B_{\alpha i}, C_{\alpha}$ are constants.

Analytically, then, our problem is to find a solution of the equation $L u=0$ subject to the boundary conditions $U_{\alpha}(u)=C_{\alpha}, \alpha=1,2, \cdots, n$. Cer-

Presented to the Society, October 29, 1949; received by the editors June 16, 1949 and, in revised form, January 7, 1950. paper.

(1) $[1$, p. 204 and p. 254]. Numbers in brackets refer to the references at the end of this 
tain additional restrictions must be placed on the initial values and on the interval $[0, c]$. These will be discussed in $\$ 1.4$.

1.3. The main results. To carry out the program outlined in $\$ 1.1$ we prove three main theorems. First, we associate with each interant $u_{m}(x)$ an "error" $\epsilon_{m}(x)$ defined by the equation $L u_{m}(x)=\epsilon_{m}(x)$. We then show the existence of two operators $M$ and $N$ such that: (i) $L=M+N$, and (ii) $\epsilon_{m+1}(x)=\left(-M N^{-}\right) \epsilon_{m}(x)$ where $N^{-}$is an appropriately defined "partial inverse" of the operator $N\left({ }^{2}\right)$. The only assumption made on the boundary conditions $U_{\alpha}(u), \alpha=1,2, \cdots, n$, needed to prove this theorem is that the differential system

$$
N u=0, \quad U_{\alpha}(u)=0, \quad \alpha=1,2, \cdots, n,
$$

is incompatible.

The second theorem, and the main one of the paper, shows that $M$ and $N$ can be so chosen that the operator $-M N^{-}$is self-adjoint and of finite norm. In order to prove this, certain additional restrictions have to be put on the boundary conditions.

Finally, in the third theorem, we show that as $m \rightarrow \infty, \epsilon_{m} \rightarrow 0$ and $u_{m}(x)$ approaches a solution of the differential system

$$
L u=0, \quad U_{\alpha}(u)=C_{\alpha}, \quad \alpha=1,2, \cdots, n .
$$

For this proof, more restrictions on the boundary conditions as well as on the length of the interval $[0, c]$ have to be made.

In short, then, we establish a transformation $T \equiv-M N^{-}$with the following properties:

(i) $T$ is self-adjoint and of finite norm.

(ii) $\epsilon_{m+1}=T \epsilon_{m}$.

(iii) $\epsilon_{m} \rightarrow 0$ as $m \rightarrow \infty$.

The operator $T$ is obtained with no restrictions on the original operator $L$ (except that of continuity of its coefficients), but involves restrictions on the boundary conditions and on the length of the interval.

The value of (i) above is seen by the following considerations. Let $\left\{\psi_{k}(x)\right\}$ be a complete orthonormal set associated with $T$ with corresponding characteristic values $\left\{\lambda_{k}\right\}$ (multiplicities included). Then if we write $\epsilon_{0}(x)=\sum_{\alpha=1}^{\infty} b_{\alpha} \psi_{\alpha}(x), \epsilon_{n}(x)=T^{n} \epsilon_{0}(x)=\sum_{\alpha=1}^{\infty} b_{\alpha} \lambda_{\alpha}^{n} \psi_{\alpha}(x)$ and $\left\|\epsilon_{n}(x)\right\|^{2}=\left(\epsilon_{n}, \epsilon_{n}\right)$ $=\sum_{\alpha=1}^{\infty} b_{\alpha}^{2} \lambda_{\alpha}^{2 n}$ where $\left\|\epsilon_{n}(x)\right\|$ is the norm of $\epsilon_{n}(x)$ and $\left(\epsilon_{n}, \epsilon_{n}\right)$ is the Hilbert space inner product. Estimates of the rapidity of convergence can be drawn from the values of the $\lambda_{\alpha}$.

The symmetry of the operator $T$ is essential for our theory. If the operator were not self-adjoint, it is possible that no expansion in characteristic functions could be made. Information on rates of convergence can be deduced from operators which, although self-adjoint, are not of finite norm. On the

(2) A construction, formally equivalent to this, is considered in $[1$, p. 263]. 
other hand, little information can be drawn from a transformation which has finite norm but which is not self-adjoint. For if $T$ has the norm $N_{r}$, then all we can say about the convergence of the $\epsilon^{\prime}$ s is that $\left\|\epsilon_{m+k}\right\| \leqq N_{T}^{k}\left\|\epsilon_{m}\right\|$. That is, we are only bounding our errors from above. In fact, since the bound, $B_{T}$, has the property $B_{T} \leqq N_{T}$ (where the $<$ sign will be assumed in general) we could use $B_{T}$ in place of $N_{T}$ to obtain a better estimate of the convergence of the $\epsilon$ 's. Thus only over estimates are available when the transformation is not self-adjoint.

1.4. Restrictions on the differential system. The restrictions on the boundary conditions necessary for Theorem 2 could be phrased in many ways. The one we have chosen seems most natural. Take any $n-1$ of the $n$ incompatible boundary conditions, say $U_{1}, U_{2}, \cdots, U_{n-1}$. Then if we can determine an $n$th boundary condition $U_{n}(u)$ such that a certain system of linear algebraic equations has a solution, then the operator $T$ is symmetric. In order to prove Theorem 3, however, a further restriction must be placed on the $U_{\alpha}(u)$ and on the interval $[0, c]$. Examples have been constructed which show that these restrictions do not lead to a vacuous theory.

1.5. Secondary results. In Theorem 4 we obtain the result that $M^{+} N$ $=N^{+} M$ where $M^{+}$and $N^{+}$are the formal or Lagrange adjoints of $M$ and $N$ respectively. This theorem is used in proving Theorem 5 , which exhibits a system of differential equations which the coefficients of the operators $M$ and $N$ satisfy. These differential equations may be of use in actually computing these coefficients.

1.6. Machine methods. One of our main interests is the application of iterative processes to mathematical machines, in particular to continuous devices. Suppose we have obtained by a continuous device an approximate solution $u_{m}(x)$ of $L u=0$ which satisfies the boundary conditions. Then we have $L u_{m}(x)=\epsilon_{m}(x)$ where $\epsilon_{m}(x)$ can be computed as accurately as desired (for example, by the use of a digital device) from the given $u_{m}(x)$. [It is important to note that any iterative process must contain an exact calculation in each step.] Now it is not difficult to invert a differential operator by machine methods. Suppose $N^{-}$has been obtained. Then $\Delta_{m}(x)$ can be computed by $\Delta_{m}=N^{-} \epsilon_{m}$ and the next iterant constructed: $u_{m+1}(x)=u_{m}(x)-\Delta_{m}(x)$. [See Theorem 1.] Scale factors can be adjusted to obtain better results at each step. The symmetry of the operator (with its real pure point spectrum) enables us to estimate the accuracy of our iterative process.

1.7. Notation. We shall use the following abbreviation for determinants whenever convenient. Let $F$ be a determinant with the element $F(i, j)$ in the $i$ th row and $j$ th column, $i, j=1,2, \cdots, n$. Then we shall write

$$
F \equiv|F(i, 1), F(i, 2), \cdots, F(i, n)|_{i=1,2, \cdots, n} .
$$

2. Preliminary lemmas. We shall establish two lemmas which will be useful in proving Theorem 2. The construction of Lemma 2 is also necessary for the 
proof of Theorem 1. First, we give a definition.

Definition 1. Let $\left\{\phi_{1}(x), \phi_{2}(x), \cdots, \phi_{n-2}(x), \Phi_{1}(x), \Phi_{2}(x)\right\}$ be $n$ linearly independent solutions of $L u=0$ where the operator $L$ has been defined in $\S 1.2$. Let $\left\{\phi_{1}(x), \cdots, \phi_{n-2}(x)\right\}$ be $n-2$ of these functions so chosen that their Wronskian $\omega(x)$ is not identically zero in $[0, c]$. Then we define the linear differential operator $M$ by the equation:

$$
\begin{aligned}
M u & =\frac{+1}{W(x)}\left|\phi_{1}^{(i)}(x), \phi_{2}^{(i)}(x), \cdots, \phi_{n-2}^{(i)}(x), u^{(i)}\right|_{i=0,1, \cdots, n-2} \\
& \equiv s_{2}(x) u^{(n-2)}+s_{3}(x) u^{(n-3)}+\cdots+s_{n}(x) u .
\end{aligned}
$$

Lemma 1. Hypothesis. Let $L$ be the linear differential operator defined in $\$ 1.2$. Let $M$ be the operator defined in Definition 1. Let $N=L-M$.

Conclusions. (i) $M$ is of order $n-2$ and $N$ is of order $n$. (ii) $L=M+N$. (iii) The Wronskian of $L$ is identical with that of $N$.

Proof. The proof is clear.

We shall now construct a "partial inverse" $N^{-}$of the differential operator $N$ using the familiar Green's function construction [1, p. 254]. This construction is valid for any incompatible linear differential system.

LEMMA 2. Hypothesis. Let

$$
N=\frac{d^{n}}{d x^{n}}+r_{1}(x) \frac{d^{n-1}}{d x^{n-1}}+\cdots+r_{n}(x) .
$$

be a linear differential operator where the $r_{i}(x)$ are real continuous functions in the closed finite interval $[0, c]$. Let $\left\{\phi_{1}(x), \phi_{2}(x), \cdots, \phi_{n}(x)\right\}$ be n linearly independent solutions of $N u=0$ and let $W(x)$ be their Wronskian. Let

(5) $g(x, \zeta)= \pm 2^{-1}(-1)^{n}\left|\phi_{i}(x), \phi_{i}(\zeta), \phi_{i}^{\prime}(\zeta), \phi_{i}^{\prime \prime}(\zeta), \cdots, \phi_{i}^{(n-2)}(\zeta)\right|_{i=1,2, \cdots, n}$ where the + sign is taken if $x \leqq \zeta$ and the - sign is taken if $x \geqq \zeta$. Let

$$
N u=0, \quad U_{\alpha}(u)=0,
$$$$
\alpha=1,2, \cdots, n \text {, }
$$

where the $U_{\alpha}(u)$ have been defined by Equation (2) be an incompatible linear differential system. Let the $a_{j}(\zeta)$ be solutions of the linear algebraic equations

$$
\sum_{i=1}^{n} a_{i}(\zeta) U_{j}\left(\phi_{i}\right)+U_{j}(g)=0, \quad j=1,2, \cdots, n .
$$

Conclusion.

$$
G(x, \zeta)=\frac{1}{W(\zeta)}\left[\sum_{i=1}^{n} a_{i} \phi_{i}(x)+g(x, \zeta)\right]
$$

is the Green's function for the system of Equations (6). 
Proof. A direct calculation shows that if $v(x)$ is any function of the class $\mathfrak{R}_{2}$ in $[0, c]$, and if

$$
u(x)=\int_{0}^{c} G(x, \zeta) v(\zeta) d \zeta
$$

then $N u=v(x)$ almost everywhere and $U_{\alpha}(u)=0, \alpha=1,2, \cdots, n$. We shall write Equation (9) symbolically as $u(x)=N^{-} v(x), U_{\alpha}(u)=0, \alpha=1,2, \cdots, n$, and the lemma shows that $N u=N N^{-} v=v$.

3. The fundamental transformation. We shall now establish the result that $\epsilon_{m}=-M N^{-} \epsilon_{m-1}$.

TheOREM 1. Hypothesis. Let the differential operator $L$ and the boundary conditions $U_{\alpha}(u), \alpha=1,2, \cdots, n$, be as defined above. Let $N$ be any differential operator of order $n$ such that

$$
N u=0, \quad U_{\alpha}(u)=0, \quad \alpha=1,2, \cdots, n,
$$

form an incompatible system, and such that

$$
L=M+N \text {, }
$$

where $M$ is an operator of order $n-2$. Let $u_{0}(x)$ be any function $\left[\in C^{n}\right]$ which satisfies the initial conditions $U_{\alpha}\left(u_{0}\right)=C_{\alpha}, \alpha=1,2, \cdots, n$. Let

$$
\begin{array}{cc}
\epsilon_{m}(x)=L u_{m}(x), \quad \Delta_{m}(x)=N^{-} \epsilon_{m}(x), & m=0,1, \cdots, \\
u_{m}(x)=u_{m-1}(x)-\Delta_{m-1}(x), & m=1,2, \cdots .
\end{array}
$$

Conclusions.

$$
\begin{aligned}
\epsilon_{m}(x) & =-M N^{-} \epsilon_{m-1}(x), & m & =1,2, \cdots, \\
U_{\alpha}\left(u_{m}\right) & =C_{\alpha}, & \alpha=1,2, \cdots, n ; m & =1,2, \cdots .
\end{aligned}
$$

Proof. Any solution of $L u=0$ has the property that it is of class $C^{n}$. [For if we assume only the existence of the $n$th derivative, the equation $f^{(n)}(x)$ $=-p_{1}(x) f^{(n-1)}(x)-\ldots-p_{n}(x) f(x)$ shows us that $f^{(n)}(x)$, where $f(x)$ is any solution of $L u=0\}$ is continuous.] Therefore there is no loss of generality in assuming the first iterant $u_{0}(x)$ to be $\in C^{n}$. Since this is the case, $\epsilon_{0}(x)$ is continuous, and hence integrable. Therefore $N^{-} \epsilon_{0}(x)$ is meaningful and $\Delta_{0}(x) \in C^{n}$. Similarly $\Delta_{1}(x), \Delta_{2}(x), \cdots \in C^{n}$. By definition of $N^{-}, U_{\alpha}\left(\Delta_{m}\right)=0$ for $m=0,1,2, \cdots$ and $\alpha=1,2, \cdots, n$. Therefore, since $u_{0}(x)$ satisfies the boundary conditions [Equation (2)], $u_{m}(x)=u_{0}(x)-\Delta_{0}(x)-\Delta_{1}(x)-\cdots$ $-\Delta_{m-1}(x)$ also satisfies them. Hence (ii) of the conclusions is satisfied.

We now prove (i). By hypothesis,

$$
\begin{aligned}
\epsilon_{m} & =L u_{m}=L u_{m-1}-L \Delta_{m-1}=\epsilon_{m-1}-(N+M) \Delta_{m-1} \\
& =\epsilon_{m-1}-N \Delta_{m-1}-M \Delta_{m-1}=-M \Delta_{m-1}
\end{aligned}
$$


since $N \Delta_{m-1}=\epsilon_{m-1}$ by Lemma 2 . Now $\Delta_{m-1}=N^{-} \epsilon_{m-1}$. Substituting this result in Equation (10) gives us

$$
\epsilon_{m}=-M N^{-} \epsilon_{m-1}\left({ }^{3}\right),
$$

which is the desired result. In general $\epsilon_{m+k}=\left(-M N^{-}\right)^{k} \epsilon_{m}$.

4. Symmetry of $M N^{-}$. We now prove our main theorem.

Theorem 2. Hypothesis. Let $M$ and $N$ be as in Lemma 1. Let $N^{-}$be the partial inverse of $N$ as constructed in Lemma 2 using the Green's function $G(x, \zeta)$. Let $U_{\alpha}(u), \alpha=1,2, \cdots, n-1$, be as defined by Equation (2). Let $\left\{\phi_{1}(x), \cdots, \phi_{n}(x)\right\}$ be $n$ linearly independent solutions of $N u=0$ with Wronskian $W(x)$.

Conclusions. If an nth boundary condition $U_{n}(u)$ can be determined such that conditions (i) and (ii) of Equation (16) below are satisfied, then the transformation $M N^{-}$can be represented as an integral operator with a real continuous symmetric kernel $F(x, \zeta)$ in the rectangle $R:\{0 \leqq x \leqq c, 0 \leqq \zeta \leqq c\}$.

Proof. By definition of $N^{-}, N^{-} f=\int_{0}^{c} G(x, \zeta) f(\zeta) d \zeta$ where $G(x, \zeta)$ is given by Equation (8). Since $M$ involves derivatives only up to and including order $n-2$, we may write $[1$, p. 257]

$$
M N^{-} f=M \cdot \int_{0}^{c} G(x, \zeta) f(\zeta) d \zeta=\int_{0}^{c} M \cdot G(x, \zeta) f(\zeta) d \zeta
$$

where it is understood that $M$ operates on functions of $x$ only. Let $F(x, \zeta)$ $=M \cdot G(x, \zeta)$ be the kernel of the integral equation (11). $F(x, \zeta)$ is obviously real and continuous. Our task is therefore to determine $U_{n}(u)$ such that $F(x, \zeta)=F(\zeta, x)$. From Equation (8)

$$
\begin{aligned}
& F(x, \zeta)=M \cdot G(x, \zeta)=\frac{1}{W(\zeta)} M \cdot\left[\sum_{i=1}^{n} a_{i}(\zeta) \phi_{i}(x)+g(x, \zeta)\right] \\
& =\frac{1}{W(\zeta)}\left[a_{n-1}(\zeta) M \phi_{n-1}(x)+a_{n}(\zeta) M \phi_{n}(x)\right]
\end{aligned}
$$

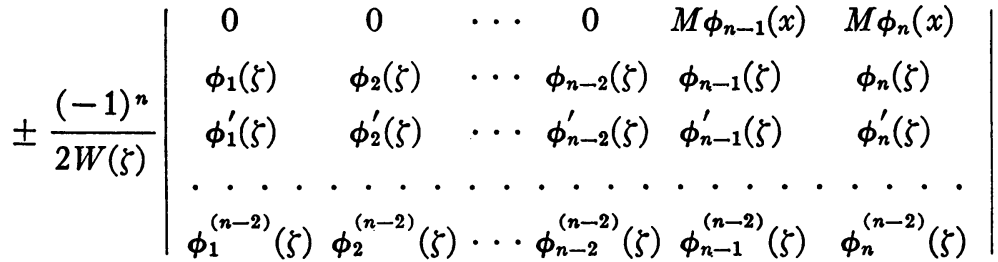

since $M \phi_{i}(x)=0$ for $i=1,2, \cdots, n-2$.

For convenience in the ensuing manipulations we introduce the notation

(3) Cf. footnote 2 . 
$W_{\alpha}^{\beta}(x)$ to mean the minor of $\phi_{\alpha}^{(\beta)}(x)$ in the Wronskian $W(x)$,

$$
W(x) \equiv\left|\phi_{1}^{(i)}(x), \phi_{2}^{(i)}(x), \cdots, \phi_{n}^{(i)}(x)\right|_{i=0,1, \cdots, n-1}
$$

and $D_{\alpha \beta}$ to mean the minor of $U_{\alpha}\left(\phi_{\beta}\right)$ in the determinant $D(U)$,

$$
D(U) \equiv\left|U_{i}\left(\phi_{1}\right), U_{i}\left(\phi_{2}\right), \cdots, U_{i}\left(\phi_{n}\right)\right|_{i=1,2}, \cdots, n \text {. }
$$

Now, Equation (12) may be written in our new notation as

$$
\begin{aligned}
F(x, \zeta)= & \frac{+1}{W(\zeta) W(x)}\left[a_{n-1}(\zeta) W_{n}^{n-1}(x)+a_{n}(\zeta) W_{n-1}^{n-1}(x)\right] \\
& \pm \frac{+1}{2 W(\zeta) W(x)}\left[W_{n-1}^{n-1}(\zeta) W_{n}^{n-1}(x)-W_{n}^{n-1}(\zeta) W_{n-1}^{n-1}(x)\right]
\end{aligned}
$$

Since in the second expression in Equation (13) the + sign is taken when $x \leqq \zeta$ and the - sign when $x \geqq \zeta$, it is evident that this expression is symmetric. It is only to render the first term of Equation (13) symmetric that the boundary conditions must be adjusted. Hence it remains to show that

$$
H(x, \zeta) \equiv a_{n-1}(\zeta) W_{n}^{n-1}(x)+a_{n}(\zeta) W_{n-1}^{n-1}(x)
$$

is symmetric. A necessary and sufficient condition that $F(x, \zeta)$ be symmetric is that $a_{n-1}(\zeta), a_{n}(\zeta)$ be such that $H(x, \zeta)=H(\zeta, x)$.

Let us express $a_{n-1}$ and $a_{n}$ in terms of the minors of $D(U)$ and $W(x)$. From Equations (7) and (5), we obtain after some straightforward calculations

$$
\begin{aligned}
a_{n} & =\frac{+1}{2 D(U)} \sum_{\beta=0}^{n-1}(-1)^{\beta} W_{\beta+1}^{n-1}(\zeta) \sum_{\alpha=1}^{n}(-1)^{\alpha-1} D_{\alpha n}\left\{V_{\alpha}\left(\phi_{\beta+1}\right)-Z_{\alpha}\left(\phi_{\beta+1}\right)\right\} \\
a_{n-1} & =\frac{-1}{2 D(U)} \sum_{\beta=0}^{n-1}(-1)^{\beta} W_{\beta+1}^{n-1}(\zeta) \sum_{\alpha=1}^{n}(-1)^{\alpha-1} D_{\alpha, n-1}\left\{V_{\alpha}\left(\phi_{\beta+1}\right)-Z_{\alpha}\left(\phi_{\beta+1}\right)\right\}
\end{aligned}
$$

We now turn to the question of determining $U_{n}$ such that $H(x, \zeta)$ is symmetric. Write

$$
a_{n}(\zeta)=\frac{+1}{2 D(U)} \sum_{\beta=0}^{n-1}(-1)^{\beta} W_{\beta+1}^{n-1}(\zeta) I_{\beta+1}
$$

where

$$
I_{\beta+1}=\sum_{\alpha=1}^{n}(-1)^{\alpha-1} D_{\alpha n}\left\{V_{\alpha}\left(\phi_{\beta+1}\right)-Z_{\alpha}\left(\phi_{\beta+1}\right)\right\}, \quad \beta=0,1, \cdots, n-1 .
$$

Now we recall that $V_{n}(u)=\sum_{i=0}^{n-1} A_{n i} u^{(i)}(0)$ and $Z_{n}(u)=\sum_{i=0}^{n-1} B_{n i} u^{(i)}(c)$. Expanding $I_{\beta+1}$ by minors of the last row, we may write: 


$$
I_{\beta+1}=\sum_{j=0}^{n-1}\left[\gamma_{j}^{\beta+1} A_{n i}+\eta_{j}^{\beta+1} B_{n i}\right], \quad \beta=0,1, \cdots, n-1 .
$$

Treating $a_{n-1}(\zeta)$ in the same manner leads to

$$
J_{\beta+1}=\sum_{j=0}^{n-1}\left[\xi_{j}^{\beta+1} A_{n j}+\theta_{j}^{\beta+1} B_{n j}\right], \quad \beta=0,1, \cdots, n-1 .
$$

Also $d(U)$, where

$$
d(U) \equiv\left|U_{i}\left(\phi_{1}\right), U_{i}\left(\phi_{2}\right), \cdots, U_{i}\left(\phi_{n-2}\right), \quad U_{i}\left(\Phi_{1}\right), U_{i}\left(\Phi_{2}\right)\right|_{i=1,2}, \cdots, n,
$$

can be written as

$$
d(U)=\sum_{j=0}^{n-1}\left[\tau_{j} A_{n j}+\rho_{j} B_{n j}\right] .
$$

The $\gamma_{j}^{\beta+1}, \eta_{j}^{\beta+1}, \xi_{j}^{\beta+1}, \theta_{j}^{\beta+1}, \tau_{j}, \rho_{j}$ are constants.

Now for symmetry we shall require:

(i)

$$
\begin{aligned}
I_{\beta+1} & =0, \\
J_{\beta+1} & =0, \\
I_{n} & =J_{n-1}, \\
d(U) & =\mu \neq 0, \\
D(U) & =\nu \neq 0 ;
\end{aligned}
$$

(ii) the rank of the augmented matrix to equal the rank of the matrix of the coefficients.

The last two conditions of (i) insure that $L u=0, U_{\alpha}(u)=0$ and $N u=0$, $U_{\alpha}(u)=0$ are both incompatible systems.

Equations (16) represent at most $2 n-1$ equations on the $2 n$ quantities $A_{n j}, B_{n j}, j=0,1, \cdots, n-1$. Now such a system of linear algebraic equations always has a solution $\left[4\right.$, p. 46]. Choose any such set of $A_{n j}^{\prime}, B_{n j}^{\prime}, j=0,1, \cdots$, $n-1$. We shall assume that

$$
U_{n}(u)=\sum_{j=0}^{n-1} A_{n j}^{\prime} u^{(j)}(0)+\sum_{j=0}^{n-1} B_{n j}^{\prime} u^{(j)}(c) .
$$

We see immediately, by virtue of Equations (16), that Equations (14) and (15) become

$$
\begin{aligned}
a_{n}(\zeta) & =\frac{+1}{2 D(U)}\left[(-1)^{n-2} W_{n-1}^{n-1}(\zeta) I_{n-1}+(-1)^{n-1} W_{n}^{n-1}(\zeta) I_{n}\right] \\
a_{n-1}(\zeta) & =\frac{-1}{2 D(U)}\left[(-1)^{n-2} W_{n-1}^{n-1}(\zeta) J_{n-1}+(-1)^{n-1} W_{n}^{n-1}(\zeta) J_{n}\right] .
\end{aligned}
$$


It is evident that $H(x, \zeta)$ is symmetric. This proves our theorem.

Let us consider the integral operator $M N^{-}$just obtained as a linear transformation in Hilbert space. We use $\mathfrak{R}_{2}$ (the class of all complex Lebesgue measurable functions which are square summable) as our realization of abstract Hilbert space. From [2, Theorem 3.8, p. 101], we see that $M N^{-}$is self-adjoint with finite norm. The desired properties of the spectrum follow from [2, Theorem 5.14, p. 193]. In particular, all points in the spectrum of $-M N^{-}$are characteristic values of finite multiplicity. Hence the arguments given in $\$ 1.3$ of the introduction are valid.

5. The existence theorem. In order to prove that $u_{m}(x)$ approaches a solution of the differential system of Equation (3) as $m \rightarrow \infty$, we shall have to place additional restrictions on the $U$ 's and on the interval $[0, c]$.

The kernel $F(x, \zeta)$ of the integral operator $M N^{-}$is a function of $D(U)$. The determinants $D(U)$ and $d(U)$ are functions of the end point $c$. Also, the coefficients $A_{n \alpha}, B_{n \alpha}, \alpha=0,1, \cdots, n-1$, are functions of the end point $c$. To indicate these relations explicitly, we write

$$
\begin{gathered}
D(U) \equiv \Delta\left[A_{n \alpha}(c), B_{n \alpha}(c), c\right], \quad d(U) \equiv \delta\left[A_{n \alpha}(c), B_{n \alpha}(c), c\right], \\
F(x, \zeta) \equiv \Omega\left[x, \zeta, A_{n \alpha}(c), B_{n \alpha}(c), c\right]
\end{gathered}
$$

where $0 \leqq x \leqq c, 0 \leqq \zeta \leqq c$. If we consider the end point $c$ as a variable, we may write

$$
\begin{gathered}
K(y, z) \equiv \Delta\left[A_{n \alpha}(y), B_{n \alpha}(y), z\right], \quad k(y, z) \equiv \delta\left[A_{n \alpha}(y), B_{n \alpha}(y), z\right], \\
\Lambda(x, \zeta, y, z) \equiv \Omega\left[x, \zeta, A_{n \alpha}(y), B_{n \alpha}(y), z\right]
\end{gathered}
$$

where the " $c$ " on which the coefficients of $U_{n}$ depend [that is, $A_{n \alpha}, B_{n \alpha}$ ] has been replaced by $y$; and where the " $c$ " on which $D(U)$ and $d(U)$ depend [that is, the " $c$ " appearing as the argument of $\phi_{\alpha}^{(\beta)}$ in the linear expressions $\left.U_{i}\left(\phi_{j}\right)\right]$ has been replaced by $z$. In particular, we note that

$$
K(c, c)=D(U), \quad k(c, c)=d(U), \quad \Lambda(x, \zeta, c, c)=F(x, \zeta) .
$$

Now, the additional and final requirements that we place on the boundary conditions and on the interval are:

$$
k(0,0) \neq 0,
$$

$$
\Lambda(0,0,0,0)<\infty .
$$

[Note that (ii) implies and is implied by $K(0,0) \neq 0$.]

We now prove a lemma (sufficient to establish Theorem 3 ) which shows the existence of an interval $\left[0, c^{*}\right], 0<c^{*} \leqq c$ with certain desirable properties.

Lemma 3. Hypothesis. Let $k(y, z)$ and $\Lambda(x, \zeta, y, z)$ as defined above have the properties that $k(0,0) \neq 0$ and $\Lambda(0,0,0,0)<\infty$.

Conclusion. There exists a $c^{*}, 0<c^{*} \leqq c$, such that for all $c^{\prime}, 0 \leqq c^{\prime} \leqq c^{*}$ :

(i) $|\Lambda(x, \zeta, y, z)| \leqq B, 0 \leqq x, \zeta, y, z \leqq c^{\prime}$ and $B$ a constant.

(ii) $c^{\prime}<1 / B$. 
(iii) $k(y, z) \neq 0,0 \leqq y, z \leqq c^{\prime}$.

Proof. Since $\Lambda$ is a continuous function of all four variables, there exists a (right-handed) neighborhood $\left[0, c_{1}\right]$ such that

$$
|\Lambda(x, \zeta, y, z)| \leqq B, \quad 0 \leqq x, \zeta, y, z \leqq c_{1},
$$

and this relation certainly holds for any non-negative values less than $c_{1}$. Since $k(y, z)$ is a continuous function, and $k(0,0) \neq 0$, there exists a (righthanded) neighborhood $\left[0, c_{2}\right]$ such that

$$
k(y, z) \neq 0, \quad 0 \leqq y, z \leqq c_{2},
$$

and this relation is also valid for any non-negative value less than $c_{2}$. Then if we define $c^{*}$ as: $c^{*}=\min \left[c_{1}, 1 / 2 B, c_{2}\right]$, this $c^{*}$ satisfies the conclusions of our lemma.

We are now in a position to state our convergence theorem.

TheOREM 3. Hypothesis. Let $M N^{-}, F(x, \zeta), \epsilon_{m}(x)$, and $u_{m}(x)$ be as defined above. Let $k(0,0) \neq 0$ and $\Lambda(0,0,0,0)<\infty$. Let $c$ satisfy the conclusions of Lemma 3.

Conclusion. The sequence of functions $u_{0}(x), u_{1}(x), \cdots$ converges uniformly to a solution $u(x)$ of the differential system of Equation (3).

Proof. We have shown in Theorem 1 that $\epsilon_{m}=-M N^{-} \epsilon_{m-1}$ and in Theorem 2 that $M N^{-} f=\int_{0}^{c} F(x, \zeta) f(\zeta) d \zeta$. Hence $\epsilon_{m}(x)=-\int_{0}^{c} F(x, \zeta) \epsilon_{m-1}(\zeta) d \zeta$. Iterating this equation yields

$\epsilon_{m+k}(x)=(-1)^{k} \int_{0}^{c} \cdots \int_{0}^{c} F\left(x, \zeta_{1}\right) F\left(\zeta_{1}, \zeta_{2}\right) \cdots F\left(\zeta_{k-1}, \zeta_{k}\right) \epsilon_{m}\left(\zeta_{k}\right) d \zeta_{1} d \zeta_{2} \cdots d \zeta_{k}$ and $\left|\epsilon_{m+k}(x)\right| \leqq H(B c)^{k}$ where $\left|\epsilon_{m}(x)\right| \leqq H$ in $[0, c]$. Since $B c<1$, the sequence $\left\{\epsilon_{m}(x)\right\}$ converges uniformly to zero.

Now by definition of the $\epsilon_{m}(x), \epsilon_{m}(x)=L u_{m}(x)$ or

$$
u_{m}(x)=\sum_{i=1}^{n-2} d_{i} \phi_{i}(x)+\sum_{i=1}^{2} e_{i} \Phi_{i}(x)+L^{-} \epsilon_{m}(x)
$$

where the $d_{i}$ and $e_{i}$ have been so chosen that $U_{\alpha}\left(u_{m}\right)=C_{\alpha}, \alpha=1,2, \cdots, n$; and where $L^{-}$is a partial inverse of $L$ based on the Green's function constructed for the incompatible system $L u=0, U_{\alpha}(u)=0, \alpha=1,2, \cdots, n$. By hypothesis, the interval $[0, c]$ was so chosen that $k(y, z)$ does not vanish in the rectangle $R:\{0 \leqq y \leqq c, 0 \leqq z \leqq c\}$, and in particular $k(c, c) \neq 0$. Hence $L^{-}$is bounded. Therefore, since the $\epsilon_{m}(x)$ converge uniformly to zero,

$$
u_{m}(x) \rightarrow \sum_{i=1}^{n-2} d_{i} \phi_{i}(x)+\sum_{i=1}^{2} e_{i} \Phi_{i}(x)
$$

uniformly. It is evident that the right-hand member of this equation is a 
solution of the differential system of Equation (3).

\section{Secondary results.}

TheOREM 4. Hypothesis. Let $L, M$, and $N$ be the linear differential operators defined above with the further restriction that $p_{1}(x), p_{2}(x), \cdots, p_{n}(x) \in C^{n}$. Let $M^{+}$and $N^{+}$be the formal or Lagrange adjoints of $M$ and $N$ respectively, that is,

$$
M^{+} u=\sum_{\alpha=2}^{n}(-1)^{n-\alpha} \frac{d^{n-\alpha}}{d x^{n-\alpha}}\left(s_{\alpha} u\right), \quad N^{+} u=\sum_{\alpha=0}^{n}(-1)^{n-\alpha} \frac{d^{n-\alpha}}{d x^{n-\alpha}}\left(r_{\alpha} u\right)
$$

where $r_{0}(x) \equiv 1, r_{1}(x) \equiv p_{1}(x)$.

Conclusion. There exists an interval $[a, b]$ contained in $[0, c]$ such that $M^{+} N=N^{+} M$ in $[a, b]$.

Proof. We shall first show that $N=P M$ where $P$ is a linear differential operator of the second order. It will then be seen that a sufficient condition for $M^{+} N$ to equal $N^{+} M$ is that $P$ be formally self-adjoint. We show that this is the case.

Now, $M u=0$ has the $n-2$ linearly independent solutions $\left\{\phi_{1}(x), \phi_{2}(x)\right.$, $\left.\cdots, \phi_{n-2}(x)\right\}$ and $N u=0$ has the $n$ linearly independent solutions $\left\{\phi_{1}(x)\right.$, $\left.\phi_{2}(x), \cdots, \phi_{n}(x)\right\}$. Let

$$
R=A(x) \frac{d^{2}}{d x^{2}}+B(x) \frac{d}{d x}+C(x)
$$

be a linear differential operator such that $R\left[M \phi_{n-1}(x)\right]=0=R\left[M \phi_{n}(x)\right]$. Then the operator $R M$ has $\left\{\phi_{1}(x), \phi_{2}(x), \cdots, \phi_{n-1}(x), \phi_{n}(x)\right\}$ as $n$ linearly independent solutions. Hence $N=h(x) R M[3$, p. 224] where $h(x) \not \equiv 0$. Let

$$
\begin{aligned}
P & =h(x) A(x) \frac{d^{2}}{d x^{2}}+h(x) B(x) \frac{d}{d x}+h(x) C(x) . \equiv h(x) R \\
& \equiv a(x) \frac{d^{2}}{d x^{2}}+b(x) \frac{d}{d x}+c(x) .
\end{aligned}
$$

where $h(x) A(x)=a(x), h(x) B(x)=b(x), h(x) C(x)=c(x)$. Then we have the result that $N=P M$ where $P$ is the linear differential operator of the second order

$$
P=a(x) \frac{d^{2}}{d x^{2}}+b(x) \frac{d}{d x}+c(x) .
$$

Now $M^{+} N=M^{+} P M$ and $N^{+} M=M^{+} P^{+} M[3$, p. 254]. Hence if we can show $P$ is formally self-adjoint, that is, $P \equiv P^{+}$, we shall have established our theorem.

$$
P^{+} u=a u^{\prime \prime}+\left(2 a^{\prime}-b\right) u^{\prime}+\left(a^{\prime \prime}-b^{\prime}+c\right) u .
$$


Comparing this with Equation (17), we see that in order for $P$ to equal $P^{+}$ it is sufficient that $a^{\prime}(x)=b(x)$. We shall prove that this is indeed the case. Writing $P M u$ in expanded form we have

$$
P M u=a s_{2} u^{(n)}+\left[a\left(2 s_{2}^{\prime}+s_{3}\right)+b s_{2}\right] u^{(n-1)}+\cdots
$$

and

$$
N u=u^{(n)}+p_{1}(x) u^{(n-1)}+r_{2}(x) u^{(n-2)}+\cdots .
$$

We can determine $a(x), b(x)$, and $c(x)$ by equating coefficients of $u^{(\alpha)}$ in $P M$ and $N$. [All but three of these relations must be redundant.]

$$
\text { From the coefficient of } u^{(n)}: a s_{2}=1 \text {. }
$$

From the coefficient of $u^{(n-1)}: a\left(2 s_{2}^{\prime}+s_{3}\right)+b s_{2}=p_{1}$.

From Equation (4) it is readily seen that

$$
\frac{d}{d x}\left[W(x) s_{2}(x)\right]=-\left[W(x) s_{3}(x)\right]
$$

Expanding this equation and remembering that the Wronskian $W(x)$ satisfies the equation $W^{\prime}+p_{1}(x) W=0$, we have

$$
s_{2}^{\prime}+s_{3}-p_{1} s_{2}=0 .
$$

In Definition 1 , the $n-2$ functions $\phi_{1}(x), \phi_{2}(x), \cdots, \phi_{n-2}(x)$ were so chosen that their Wronskian $\omega(x) \not \equiv 0$. Since $\omega(x)$ is continuous, there exists a point and a neighborhood of this point such that $\omega(x) \neq 0$ in this neighborhood. Let this region be the $[a, b]$ interval (a subset of $[0, c]$ ) referred to in the statement of the theorem.

Multiply Equation (19) by $s_{2}$, replace $a s_{2}$ by 1 by virtue of Equation (18) and subtract Equation (20). There results $s_{2}^{\prime}+b s_{2}^{2}=0$ or $b=-s_{2}^{\prime} / s_{2}^{2}$. But from Equation (18), $a=1 / s_{2}$. Hence $a^{\prime}=b$, as was desired to be proved.

Theorem 5. Hypothesis. Let $M, N, M^{+}, N^{+}$be as in Theorem 4. Let $X_{\alpha \beta}=s_{\alpha}(x) r_{\beta}(x)-r_{\alpha}(x) s_{\beta}(x) \equiv s_{\alpha} p_{\beta}-p_{\alpha} s_{\beta}, \quad \alpha, \beta=0,1, \cdots, n$ where $s_{0}(x)$ $=s_{1}(x) \equiv 0$ and $r_{0}(x) \equiv 1, r_{1}(x) \equiv p_{1}(x)$.

Conclusion. In the interval $[a, b]$

$$
q_{j}(x)=\sum_{k=2}^{j} \sum_{\alpha=0}^{k}(-1)^{-\alpha} C_{n-\alpha, n+k-j-\alpha} X_{\alpha, k-\alpha}^{(j-k)} \equiv 0, \quad \alpha \leqq n,
$$

$j=3,4, \cdots, 2 n$, where $C_{\alpha,-\beta}$ is regarded as zero for $\alpha, \beta>0$.

Proof. Consider the expression $Q u \equiv\left(M^{+} N-N^{+} M\right) u$. From Theorem 4, $Q u \equiv 0$ in $[a, b]$. We shall calculate the coefficients of $u^{(\alpha)}$ in $Q u$. They will be the $q_{j}(x)$ functions mentioned in the theorem. Necessarily, they must all be identically zero. While $q_{j}(x)=0, j=3,4, \cdots, 2 n$, represent $2 n-2$ equations, 
actually only $n-1$ are independent since they are differential equations on the $n-1$ functions $s_{2}(x), s_{3}(x), \cdots, s_{n}(x)$. [By dependent we mean that some $q_{j}(x)$ can be expressed as a linear combination of some other of the $q_{i}(x)$ and their derivatives, with constant coefficients.] We shall compute the $q_{j}(x)$. Now

$$
Q u=M^{+} N u-N^{+} M u=\sum_{\alpha=0}^{n} \sum_{\beta=0}^{n}(-1)^{n-\alpha} \frac{d^{n-\alpha}}{d x^{n-\alpha}}\left\{\left[s_{\alpha} r_{\beta}-r_{\alpha} s_{\beta}\right] \frac{d^{n-\beta} u}{d x^{n-\beta}}\right\} .
$$

Introducing $X_{\alpha \beta}(x)$ and noting that $[f(x) g(x)]^{(m)}=\sum_{\mu=0}^{m} C_{m, \mu} f^{(\mu)} g^{(m-\mu)}$ where $C_{m, \mu}$ is the binomial coefficient, $Q u$ becomes

$$
Q u=\sum_{\alpha=0}^{n} \sum_{\beta=0}^{n}(-1)^{n-\alpha} \sum_{\gamma=0}^{n-\alpha} C_{n-\alpha, \gamma} X_{\alpha \beta}^{(n-\alpha-\gamma)} u^{(n-\beta+\gamma)} .
$$

Let $j=n-\gamma+\beta$. Then

$$
Q u=\sum_{\alpha=0}^{n} \sum_{\beta=0}^{n} \sum_{j=\alpha+\beta}^{n+\beta}(-1)^{n-\alpha} C_{n-\alpha, n+\beta-j} X_{\alpha \beta}^{(i-\alpha-\beta)} u^{(2 n-j)} .
$$

Let

$$
Q u=(-1)^{n}\left[q_{0}(x) u^{(2 n)}+q_{1}(x) u^{(2 n-1)}+\cdots+q_{2 n}(x) u\right]
$$

be the defining equation for the $q_{j}(x)$. We see that the coefficient of $(-1)^{n} u^{(2 n-j)}$ is $q_{j}(x)$. From Equation (21)

$$
q_{j}(x)=\sum_{k=0}^{j} \sum_{\alpha=0}^{k}(-1)^{\alpha} C_{n-\alpha, n+k-j-\alpha} X_{\alpha, k-\alpha,}^{(j-k)} \quad \alpha \leqq n,
$$

for all $j=0,1, \cdots, 2 n$, with the proviso that $n+k \geqq \alpha+j$. We note that the denominator of the binomial coefficient in Equation (22) is precisely $n+k$ $-(j+\alpha)$. So if we adopt the convention that the binomial coefficient $C_{\alpha,-\beta}$, $\alpha, \beta>0$, is defined as zero, Equation (22) is correct as it stands with no qualifying statement.

Due to introducing $s_{0}(x)$ and $s_{1}(x)$ (which are actually zero), we may write Equation (22) with the summation extending from $k=2$ to $j$ instead of from $k=0$ to $j$, since these cases are vacuous.

\section{REFERENCES}

1. E. L. Ince, Ordinary differential equations, Dover Publications, New York, 1944.

2. M. H. Stone, Linear transformations in Hilbert space, Amer. Math. Soc. Colloquium Publications, vol. 15, 1932.

3. A. R. Forsyth, Theory of differential equations, part 3, vol. 4, Cambridge University Press, 1902.

4. M. Bôcher, Introduction to higher algebra, Macmillan Company, New York, 1907.

Columbia University, New York, N. Y. 